\title{
The effect of implementing nursing practices to support ventilation among patients with acute respiratory failure on their condition
}

\author{
Mervat Anwar AbdEl-Aziz ${ }^{1}$, Manal Saad Shaker Soliman² \& Mona Mohamed Ibrahim ${ }^{3 .}$ \\ 1. Assistant Professor of Critical Care \& emergency nursing, Faculty of Nursing, Assiut University, Assiut, Egypt. \\ 2. Lecturer, Medical Surgical Nursing, Faculty of Nursing, Fayoum University, Egypt. \\ 3. Lecturer, Medical Surgical Nursing, Faculty of Nursing, Beni_Seuif University, Egypt.
}

\begin{abstract}
Background: Acute respiratory failure is a sudden and life-threatening deterioration in pulmonary gas exchange, resulting in carbon dioxide retention and inadequate oxygenation approximately $50 \%$ to $75 \%$ of those patients require ICU hospital stays \&and mechanical ventilatory support practices longer than 7 days. Aim: This study aimed to evaluate the effect of implementing nursing practices to support ventilation among patients with acute respiratory failure on their condition. Method: Design: A quasi-experimental research design was used to conduct this research. A convenient sample consisted of 120 patients, they were enrolled in the study group $(n=60)$ and the control group $(\mathrm{n}=60)$ was admitted to the chest intensive care unit at Assiut University Hospital. A patient assessment sheet, acute respiratory failure Patient outcomes assessment sheet were used for the collection of data. Results: A significant statistical difference $(\mathrm{P}<0.05)$ between both groups concerning the improvement of oxygenation, arterial blood gas, clinical pulmonary infection score, length of chest ICU stay, mechanical ventilator parameters among study group compared to control group subjects after the intervention, thus research hypotheses cane bee supported. Conclusion: Implementing nursing practices to support ventilation among patients with acute respiratory failure effective in improving their outcomes. Recommendation: develop standardized nursing guidelines for patients with acute respiratory failure.
\end{abstract}

\section{Keywords: Outcomes, Practices, Respiratory Failure \&Ventilatory Support}

\section{Introduction}

Respiratory failure is a syndrome of inadequate gas exchange which involves the transfer of $\left(\mathrm{O}_{2}\right)$ and carbon dioxide $\left(\mathrm{Co}_{2}\right)$ between the atmosphere and the blood is inadequate. Moreover, respiratory failure is not a disease it is a condition that occurs as a result due to dysfunction of one or more essential compound of the respiratory center include chest wall diseases, pulmonary circulation, airway obstruction, or parenchymal lung disorders, abnormal respiratory neuromuscular system, CNS or brain stem, alveolarcapillary units Alcibey \& Isabel (2016).

Acute respiratory failure caused by a variety of pulmonary and no pulmonary diseases usually defined by an arterial oxygen tension ( $\mathrm{PaO} 2)$ of less than $60 \mathrm{mmHg}$ and/or an arterial carbon dioxide tension $(\mathrm{PaCO} 2)$ greater than $45 \mathrm{mmHg}$ Struik et al., (2018) . Acute respiratory failure is classified as an acute hypoxemic respiratory failure (type I), acute hypercapnic respiratory failure (type II), or combined hypoxemic and hypercapnic respiratory failure (type I and type II). Hypoxemia and hypercapnia affect every organ and tissue and impairs cellular functions, which include the following: Increased pulmonary vascular resistance, cor pulmonale, Right-sided heart failure, impaired left ventricular function, reduced cardiac output, cardiogenic pulmonary edema, Diaphragmatic fatigue from the increased workload of respiratory muscles Struik et al., (2018). Treatment of acute respiratory failure warrants immediate intervention to correct or compensate for the gas exchange abnormality in the intensive care unit (ICU) and identify the cause toward correcting the cause and alleviating the hypoxia and hypercapnia correct. Although the recommended therapeutic intervention endotracheal intubation and mechanical ventilation may be lifesaving to minimize the life-threatening complications associated with extended hypoxemia increase $\mathrm{PaO} 2$ by increasing $\mathrm{FIO} 2$ and to normalize $\mathrm{pH}$ by increasing minute ventilation Elizabeth \& Deborah (2018). Bronchodilators, corticosteroids, antibiotics and supplemental oxygen should also be initiated and titrated to achieve an oxygen saturation of $88 \%$ to $92 \%$. As an alternative to oxygen therapy, oxygen via a high-flow nasal cannula or noninvasive positive pressure ventilation can also be used to improve oxygenation and ventilation and decrease hypercapnia in acute hypoxemic respiratory failure Mirza et al., (2018). Critical care nurse must be assessing the patient's chest radiograph for pulmonary findings to determine which regions of the lung require attention, before implementing chest physiotherapy procedure, assess the patient respiratory rate, rhythm, breath sound by lung auscultation at least every 2 to 4 hours, dyspnea, skin color, sputum production including 
(color, quantity, consistency, color, odor) mental status and oxygen saturation by a pulse oximeter, monitor hemodynamic parameters as heart rate, blood pressure Kaplow\& Hardine (2012).

Mechanically ventilated patients should be turned at least every two hours as tolerated. Posture drainage: during the acute phase of a pulmonary process, perform chest physiotherapy at least every two-hour, percussion-vibration: perform as needed in conjunction with posture drainage Nancy \& cliesla (2014) .Encourage fluid intake to encourage intake of protein and caloric rich foods, encourage coughing and deep breathing, assess pain characteristics (quality, severity, location, onset, duration, and relieving factors) Lea et al., (2016) Assess effective airway clearance for patency, inspect the mouth, neck, and position of the trachea for potential obstruction, perform measures to clear the airway of secretions as (suctioning, frequent position change ), suctioning should be performed, auscultate lungs for the presence of normal or adventitious lung sounds as wheezing which indicates airway resistance, stridor indicates emergent airway obstruction Gil Wayne, (2016) The role of critical care nurse is to assess respirations as note quality, rate, pattern, depth, and breathing effort, assess lung sound, presence of adventitious sounds, assess for signs and symptoms of hypoxemia (tachycardia, restlessness, diaphoresis, headache, lethargy, and confusion) monitor vital signs, assess for changes in orientation and behavior, monitor arterial blood gases $\left(\mathrm{ABG}_{\mathrm{S}}\right)$, monitor chest $\mathrm{X}$ ray reports, assess color and consistency of sputum Meg \& Judith (2017).

\section{Significance of the study}

Acute respiratory failure (ARF) in critically ill patients is still accompanying mortality rates of between $40 \%$ and $65 \%$. Most of the available literature has attentive on the severest form of ARF, namely, ARDS, and scarcely studied have weighed either the incidence of the mortality rate of ARF in general between critically ill patients Culnan, et al., (2018) Statistics of Intensive care unit at Assiut University Hospital admitted to Chest intensive care unit is ranged 1500 patients in the years of (2019) approximately $75 \%$ of them diagnosed Acute respiratory failure.By observation of the research found that occurrences of complications especially VAP is the most common nosocomial infection in ICU with high morbidity and mortality rate. It is also observed to be considerably contributing factor to increase the length of hospital stay. The purpose of this research study is to show the effectiveness of using different modalities of chest physiotherapy on the prevention of VAP, a complication linked with prolonged MV. Improved outcomes will shorten patients' ICU and length of stay.

Aim of the study

This study aimed evaluate effect of implementing nursing practices to support ventilation among patients with acute respiratory failure on their condition.

\section{Patients and method}

\subsection{Design:}

A quasi-experimental research design was used to conduct this research

2.2. Hypotheses: To fulfill the aim of the study the following research hypotheses were formulated:

Hypotheses (1) the expected mean respiratory assessment value of the study group will be better than those of the control group: -

Hypotheses (2) the post mean hemodynamic parameters of the study group will be better than those of the control group.

Hypotheses (3) the expected mean arterial blood gas value of the study group will be better than those of the control group.

Hypotheses (4) the post mean clinical pulmonary infection reading of patients in the study group will be better than those of the control group.

Hypotheses (5) the post means patients outcomes reading of patients on the study group will be better than those of the control group.

\subsection{Setting}

The study was conducted at the chest intensive care unit at Assiut University Hospital.

\subsection{Subjects}

A convenient sample consisted of 120 critically ill adult patients (male and female) who were admitted to the chest intensive care unit and the diagnosis of acute respiratory failure, Connected to a mechanical ventilator. They were randomly assigned into two equal groups 60 patients (control group \& study group).

\subsection{Study Tools}

Three tools were utilized to collect data in this study. They are developed by the researcher after passing through an extensive and relevant review of the literature. The validity \& reliability of these tools were revised by a panel of critical care nursing and medical experts, and then a pilot study was done.

Tool I: Acute respiratory failure patient assessment sheet: -

(Higginson \&Jones et al., 2009' Ames et al., 2011)

The assessment sheet was developed by the researcher after extensive literature of review. It composed of 6 main areas

1- Socio-demographic data and clinical data (age, sex) type acute respiratory failure

2- Respiratory system assessment in terms of the following (breath sounds,) and (Amount of 
tracheal secretions, the character of tracheal secretion)

3- Ventilator profile (mode of ventilation, pressure support (PS) ,tidal volume (vt) ,respiratory rate (f),fraction of inspired oxygen (fio2), positive end-expiratory pressure (PEEP)

4- Assessment of hemodynamic parameters(temperature, heart rate, respiratory rate and blood pressure (systolic \& diastolic) and MAP)

5- laboratory investigation Arterial blood gases, assessment of sputum culture to detect infection, $\mathrm{X}$ - chest radiograph, white blood cells count

6- Assessment of Clinical pulmonary infection score (CPIS)to detect the presence or absence of pneumonia

Tool 11- Acute respiratory failure Patient's outcomes assessment sheet,

This tool designing to study $\mathbf{2}$ main areas:

a- Acute respiratory failure complication as (pneumonia, renal failure, Congestive heart failure, pulmonary edema, pulmonary embolism, pneumothorax, and abdominal distention)

b- Expected patient's outcomes as ( mortality rate, duration of mechanical ventilation, duration of ICU stays, length of chest ICU stay

\subsection{Methods}

This study where carried out through three main phases as follows

\section{The preparatory phase}

- An official Permission to conduct the study was obtained from the hospital responsible authorities in the chest intensive care unit at Assiut University Hospital after explaining the aim and nature of the study.

- Content validity: The tools were tested for content related validity by a jury of 6 specialists in the field of critical care nursing and critical care medicine from the chest care unit at Assiut University Hospital and the necessary modification was done.

\section{Pilot study}

A pilot study carried out before starting of data collection to test the applicability and clarity of the study tools on $10 \%$ the study sample, the purpose of the pilot study was: to ascertain the relevance of the tool, detect any problem might interfere with the process of data collection and to estimate the time needed to complete the study, define the modification required in the tool used, and the necessary modification was done before data collection, the studied subjects were excluded from the actual study.

\section{Ethical considerations}

- Approval was obtained from the local ethical committee and the study was followed the common ethical principles in clinical research.
- The tool used in this study was developed based on reviewing the relevant literature.

- Protection of human rights (ethical considerations): Informed consent was obtained from each patient or the responsible person for the unconscious patients. The investigator emphasized that participation is voluntary and the confidentiality and anonymity of the subjects were assured through coding the data. Subjects were assured that can they withdraw from the study at any time without any rationale.

\section{Data collection}

Started from the first of February 2019 until February 2020. The data were collected from the first day of admission after stabilization of the patient's condition consequent days, every day then the data were recorded in the developed tools.

\section{Implementation phase}

- The studied sample fulfilling the research criteria was randomly assigned into two groups (study group and control group).

- Assessment of both groups by research from patient files for the following data (Sociodemographic data,

$\checkmark$ The researcher assessed hemodynamic state including (temperature $(\mathrm{T})$, blood pressure $(\mathrm{Bb})$, heart rate (HR), respiratory rate and mean arterial pressure (MAP) was recorded for seven consequent days

$\checkmark$ The respiratory state was assessed for breath sound which includes: (normal, crackles, Ronchi, and wheezing) and lung tracheal secretion was done to detect any abnormalities.

$\checkmark$ Assess ventilator profile mode of ventilation, pressure support (PS), tidal volume (vt), respiratory rate (f), a fraction of inspired oxygen (fio2), positive end-expiratory pressure (PEEP)

$\checkmark$ Assessment of arterial blood gases (ABG): (PH, $\mathrm{Pao} 2, \mathrm{Paco} 2, \mathrm{Hco} 3$ and oxygen saturation )

$\checkmark$ Sputum culture of the respiratory secretion was taken early in the morning, which done to provide baseline data

$\checkmark$ Measurement of clinical pulmonary infection score (CPIS) was used to detect the presence or absence of pneumonia. It consists of five items including (temperature, white blood cells count, the character of tracheal secretion, infiltrates on $\mathrm{X}$ - chest radiograph and $\mathrm{PaO}_{2}$ of inspired oxygen) each item takes a score from 0 to 2 for each item. If the total score is equal or more than (6) suspected pneumonia but if it is less than (6) it means no presence of pneumonia. (Zilberberg \& shorr, 2010).

$\checkmark$ Monitor the patient outcomes, assess occurrence of acute respiratory failure patient complication as (pneumonia, renal failure, Congestive heart 
failure, pulmonary edema, pulmonary embolism, pneumothorax, and abdominal distention) during chest ICU stay, number of days in mechanical ventilator, ICU duration, and mortality rate

The control group: -received the routine hospital care for critically ill mechanically ventilated patients without intervention from the researcher.

\section{The study group}

The patient received the developed nursing care for acute respiratory failure mechanically ventilated patients as follows

$\checkmark$ Chest physiotherapy from the first day of admission until the seventh day of the study.

$\checkmark$ Each patient was placed in a baseline semi setting position with the elevated head of the bed 30 degrees for 10 minutes before the commencement of the study.

$\checkmark$ The measurement of hemodynamic parameters, blood gases, and auscultator findings was done to determine breath sounds or any abnormal sounds before starting and after 2 hours of chest physiotherapy.

$\checkmark$ Each postural drainage position was maintained for 5 minutes and modified according to the patient's condition

$\checkmark$ Percussion and vibration were applied followed by suction using a sterile technique. The patient was pre-oxygenated with $100 \%$ before suction to prevent desaturation.

$\checkmark$ During turning the patient any tubes and connections attached to the patient were observed as ECG monitor, feeding tube, urinary catheter, the arterial line to avoid pulling, stretching, or kilning these tubes were avoided.

$\checkmark$ Oxygen saturation and heart rate were monitoring using pulse Oximetry

$\checkmark$ Patients have received $5 \mathrm{~mL}$ of $0.9 \%$ saline 4 times/day alternately with bronchodilator according to physician's order which given 1530 minutes before chest physiotherapy.

$\checkmark$ For a non-invasive ventilated patient, (Perform deep breathing, coughing exercise every 4 hours, administration of oxygen via a Venturi mask or nasal cannula To Increase the inspired oxygen (FIO2) concentration

$\checkmark$ Monitor breath sounds for crackles, wheezes, or rhonchi every $2-4 \mathrm{~h}$.

$\checkmark$ Administer diuretics as order.

$\checkmark$ Monitor fluid intake and output closely.

$\checkmark$ Monitor respiratory rate, pattern, and effort (eg, use of accessory muscles)

$\checkmark$ Correct $\mathrm{pH}$ disturbances: In acute hypercapnia with acidosis, improve alveolar ventilation by establishing and maintaining an adequate airway, treating bronchospasm, and controlling heart failure, fever, and sepsis. $\checkmark$ Consider bicarbonate administration in acute respiratory acidosis or metabolic acidosis

$\checkmark$ Perform daily body weight measurement.

$\checkmark$ Prevent and treat promptly hypokalemia and hypophosphatemia.

$\checkmark$ Maintain adequate cardiac output.

$\checkmark$ Prevent and treat abdominal distension by insertion of a nasogastric tube

$\checkmark$ Control fever and other causes of increased metabolism

$\checkmark$ Assess pain/comfort every $4 \mathrm{~h}$. Administer analgesics and sedatives cautiously, closely monitoring respiratory rate, depth, and pattern.

$\checkmark$ Provide parenteral, enteral, or oral nutrition within $48 \mathrm{~h}$

$\checkmark$ Assess risk for skin breakdown using an objective tool (eg, Braden scale). Consider pressure relief/reduction mattress

$\checkmark$ Promote standing at the bedside, sitting up in a chair, ambulating with assistance as soon as possible.

$\checkmark$ Use a passive and active range of motion every $4 \mathrm{~h}$ while awake.

$\checkmark$ Monitor systemic inflammatory response syndrome criteria: increased WBC count, increased temperature, tachypnea, and tachycardia.

$\checkmark$ Use strict aseptic technique during procedures

$\checkmark$ Assess for signs of right ventricular dysfunction (eg, increased central venous pressure, neck vein distention, peripheral edema).

$\checkmark$ Encourage nonintubated patients to use an incentive spirometer, cough, and deep breathe every $2-4 \mathrm{~h}$.

$\checkmark$ At the end of the procedure leaving the patient to the routine nursing intervention.

\section{Evaluation phase}

Both groups were evaluated daily using tool two $\left(1^{\text {st }}\right.$ part and $2^{\text {nd }}$ part) hemodynamic (temperature, heart rate, and respiratory rate).

-Respiratory \& ventilator parameters assessment daily to determine the effect of implementing nursing care on patients with acute respiratory failure outcomes.

- Clinical pulmonary infection score assessment daily to determine the effect of implementing nursing care on infection control for ventilated patients associated pneumonia.

- Evaluate patient's outcomes which consist of (duration of mechanical ventilation, length of ICU stay, mortality rate and respiratory failure patient complication) 


\section{Statistical analysis}

All data were recorded on a special chart for every patient. The collected data were coded, analyzed, and tabulated. Data entry and analysis were done using SPSS 17.0 statistical software package. Data were presented using descriptive statistics in the form of frequencies and percentages for qualitative variables, and means and standard deviations for quantitative variables. Quantitative continuous data were compared using analysis of variance test in case of comparisons between two independent groups. Using chi-square test for non-parametric data to determine statistical significant differences were considered when P-value used as follows: - P >0.05 nonsignificant $* \mathrm{P}<0.05$ significant $* * \mathrm{P}<0.01$ moderate significant $* * * \mathrm{P}<0.001$ highly significant

\section{Results}

Table (1): Percentage distribution of studied patients regarding demographic characteristics and clinical $\operatorname{data}(n=120)$.

\begin{tabular}{|c|c|c|c|c|c|}
\hline \multirow{2}{*}{ Variables } & \multicolumn{2}{|c|}{ Study $(n=60)$} & \multicolumn{2}{|c|}{ Control $(n=60)$} & \multirow{2}{*}{ P-value } \\
\hline & No. & $\%$ & No. & $\%$ & \\
\hline \multicolumn{5}{|l|}{ Age: (years) } & \multirow{4}{*}{0.818} \\
\hline$<40$ & 26 & 43.3 & 27 & 45.0 & \\
\hline $40-<50$ & 26 & 40.0 & 21 & 35.0 & \\
\hline$\geq 50$ & 10 & 16.7 & 12 & 20.0 & \\
\hline Mean \pm SD & \multicolumn{2}{|c|}{$47.16 \pm 17.38$} & \multicolumn{2}{|c|}{$46.74 \pm 15.39$} & 0.890 \\
\hline \multicolumn{5}{|l|}{ Sex: } & \multirow{3}{*}{0.852} \\
\hline Male & 37 & 61.7 & 36 & 60.0 & \\
\hline Female & 23 & 38.3 & 24 & 40.0 & \\
\hline \multicolumn{5}{|l|}{ Type respiratory failure } & \multirow{3}{*}{0.084} \\
\hline Type I hypoxemic & 18 & 30.0 & 10 & 16.7 & \\
\hline Type II hypercapnic & 42 & 70.0 & 50 & 83.3 & \\
\hline \multicolumn{5}{|l|}{ type of Admission ICU: } & \multirow{3}{*}{0.855} \\
\hline First time & 31 & 51.7 & 30 & 50.0 & \\
\hline Recurrent & 29 & 48.3 & 30 & 50.0 & \\
\hline
\end{tabular}

Ns: There is no significant difference $(p>0.05)$

Chi-square test Independent samples t-test

Hypothesis 1, the expected mean respiratory assessment value of the study group will be better than those of the control group, as (breath sound, tracheal secretion Table (2,3):

Table (2): Percentage distribution of the studied patients regarding breath sound

\begin{tabular}{|c|c|c|c|c|c|c|}
\hline & \multirow{2}{*}{ Breath sound } & \multicolumn{2}{|c|}{ Study $(n=60)$} & \multicolumn{2}{|c|}{ Control $(n=60)$} & \multirow{2}{*}{ P-value } \\
\hline & & No. & $\%$ & No. & $\%$ & \\
\hline \multirow{4}{*}{$1^{\text {st }}$ day } & Normal & 0 & 0.0 & 0 & 0.0 & \multirow{4}{*}{0.181} \\
\hline & Crackles & 3 & 5.0 & 0 & 0.0 & \\
\hline & Wheezes & 15 & 25.0 & 13 & 21.7 & \\
\hline & Ronchi & 42 & 70.0 & 47 & 78.3 & \\
\hline \multirow{4}{*}{$2^{\text {nd }}$ day } & Normal & 0 & 0.0 & 0 & 0.0 & \multirow{4}{*}{0.190} \\
\hline & Crackles & 1 & 1.7 & 3 & 5.0 & \\
\hline & Wheezes & 17 & 28.3 & 12 & 20.0 & \\
\hline & Ronchi & 42 & 70.0 & 45 & 75.0 & \\
\hline \multirow{4}{*}{$3^{\text {rd day }}$} & Normal & 5 & 8.3 & 2 & 3.3 & \multirow{4}{*}{0.449} \\
\hline & Crackles & 2 & 3.3 & 4 & 6.7 & \\
\hline & Wheezes & 15 & 25.0 & 18 & 30.0 & \\
\hline & Ronchi & 38 & 63.3 & 36 & 60.0 & \\
\hline \multirow{4}{*}{$4^{\text {th }}$ day } & Normal & 35 & 58.3 & 10 & 16.7 & \multirow{4}{*}{$0.045^{*}$} \\
\hline & Crackles & 5 & 8.3 & 9 & 15.0 & \\
\hline & Wheezes & 12 & 20.0 & 17 & 28.3 & \\
\hline & Ronchi & 8 & 13.3 & 24 & 40.0 & \\
\hline $7^{\text {th }}$ day & Normal & 43 & 71.7 & 15 & 25.0 & $0.005^{*}$ \\
\hline
\end{tabular}




\begin{tabular}{|l|c|c|c|c|c|c|}
\hline \multirow{2}{*}{ Breath sound } & \multicolumn{2}{|c|}{ Study $(\mathbf{n}=\mathbf{6 0})$} & \multicolumn{2}{c|}{ Control (n= 60) } & \multirow{2}{*}{ P-value } \\
\cline { 2 - 6 } & No. & $\mathbf{\%}$ & No. & \% \\
\cline { 2 - 6 } & Crackles & 2 & 3.3 & 8 & 13.3 \\
\cline { 2 - 6 } & Wheezes & 8 & 13.3 & 18 & 30.0 \\
\cline { 2 - 6 } & Ronchi & 7 & 11.7 & 19 & 31.7 & \\
\hline
\end{tabular}

Ns: There is no significant difference $(P>0.05) *$ Statistically significant difference $(p<0.05)$

Chi-square test

Table (3): percentage distribution of the studied patients regarding the amount of tracheal secretion

\begin{tabular}{|c|c|c|c|c|c|c|}
\hline & \multirow{2}{*}{ Amount of tracheal secretion } & \multicolumn{2}{|c|}{ Study $(n=60)$} & \multicolumn{2}{|c|}{ Control $(n=60)$} & \multirow{2}{*}{ P-value } \\
\hline & & No. & $\%$ & No. & $\%$ & \\
\hline \multirow{3}{*}{$1^{\text {st }}$ day } & \begin{tabular}{|l|} 
Small \\
\end{tabular} & 49 & 81.7 & 58 & 96.7 & \multirow{3}{*}{$0.008^{*}$} \\
\hline & Moderate & 11 & 18.3 & 2 & 3.3 & \\
\hline & High & 0 & 0.0 & 0 & 0.0 & \\
\hline \multirow{3}{*}{$2^{\text {nd }}$ day } & \begin{tabular}{|l|} 
Small \\
\end{tabular} & 20 & 33.3 & 40 & 66.6 & \multirow{3}{*}{0.225} \\
\hline & Moderate & 35 & 58.3 & 13 & 21.7 & \\
\hline & High & 8 & 13.3 & 7 & 11.7 & \\
\hline \multirow{3}{*}{$3^{\text {rd day }}$} & Small & 8 & 13.3 & 39 & 65.0 & \multirow{3}{*}{$0.010 *$} \\
\hline & Moderate & 42 & 70.0 & 12 & 20.0 & \\
\hline & High & 10 & 16.7 & 9 & 15.0 & \\
\hline \multirow{4}{*}{$4^{\text {th }}$ day } & Free & 35 & 58.3 & 12 & 20.0 & \multirow{4}{*}{$0.017 *$} \\
\hline & Small & 18 & 30.0 & 19 & 31.7 & \\
\hline & Moderate & 5 & 8.3 & 19 & 31.7 & \\
\hline & High & 2 & 3.3 & 10 & 16.6 & \\
\hline \multirow{4}{*}{$7^{\text {th }}$ day } & Free & 42 & 70.0 & 20 & 33.3 & \multirow{4}{*}{$0.006^{*}$} \\
\hline & \begin{tabular}{|l|} 
Small \\
\end{tabular} & 8 & 13.3 & 23 & 38.3 & \\
\hline & \begin{tabular}{|l|} 
Moderate \\
\end{tabular} & 8 & 13.3 & 10 & 16.7 & \\
\hline & High & 2 & 3.3 & 7 & 11.7 & \\
\hline
\end{tabular}

Ns: There is no significant difference $(P>0.05) *$ Statistical significant difference $(p<0.05)$

Chi-square test

Hypotheses (2) the post mean hemodynamic parameters of the study group will be better than those of the control group

Table (4): Hemodynamic parameters mean values for the studied patients.

\begin{tabular}{|c|c|c|c|c|}
\hline & \multirow{2}{*}{$\begin{array}{c}\text { Hemodynamic } \\
\text { parameters }\end{array}$} & \multirow{2}{*}{$\begin{array}{c}\text { Study }(n=60) \\
\text { Mean } \pm \text { SD }\end{array}$} & \multirow{2}{*}{$\begin{array}{c}\text { Control }(n=60) \\
\text { Mean } \pm \text { SD }\end{array}$} & \multirow{2}{*}{ P-value } \\
\hline & & & & \\
\hline \multirow{6}{*}{$1^{\text {st }}$ day } & Heart rate & $90.77 \pm 12.03$ & $96.82 \pm 17.99$ & $0.032 *$ \\
\hline & Respiratory rate & $24.23 \pm 3.53$ & $24.85 \pm 3.39$ & 0.331 \\
\hline & \begin{tabular}{|l|} 
Temperature \\
\end{tabular} & $37.30 \pm 0.30$ & $37.36 \pm 0.27$ & 0.279 \\
\hline & \begin{tabular}{|l} 
Systolic BP \\
\end{tabular} & $122.67 \pm 16.04$ & $126.33 \pm 14.14$ & 0.187 \\
\hline & Diastolic BP & $87.00 \pm 82.30$ & $79.50 \pm 9.10$ & 0.484 \\
\hline & MAP & $84.50 \pm 15.59$ & $87.92 \pm 11.87$ & 0.179 \\
\hline \multirow{6}{*}{$2^{\text {nd }}$ day } & Heart rate & $93.47 \pm 12.52$ & $99.23 \pm 18.47$ & $0.049^{*}$ \\
\hline & \begin{tabular}{|l} 
Respiratory rate \\
\end{tabular} & $23.70 \pm 3.35$ & $28.34 \pm 3.42$ & $0.003^{*}$ \\
\hline & Temperature & $37.12 \pm 0.29$ & $39.34 \pm 0.29$ & $0.000^{*}$ \\
\hline & \begin{tabular}{|l} 
Systolic BP \\
\end{tabular} & $120.33 \pm 15.40$ & $126.95 \pm 14.17$ & $0.016^{*}$ \\
\hline & Diastolic BP & $74.02 \pm 9.24$ & $80.34 \pm 9.82$ & $0.000^{*}$ \\
\hline & \begin{tabular}{|l|} 
MAP \\
\end{tabular} & $82.92 \pm 15.05$ & $88.47 \pm 12.19$ & $0.029 *$ \\
\hline \multirow{3}{*}{$3^{\text {rd }}$ day } & Heart rate & $95.11 \pm 13.93$ & $99.69 \pm 16.89$ & $0.031^{*}$ \\
\hline & Respiratory rate & $25.83 \pm 4.02$ & $28.70 \pm 3.31$ & $0.031 *$ \\
\hline & Temperature & $38.38 \pm 0.05$ & $39.44 \pm 0.30$ & $0.011^{*}$ \\
\hline
\end{tabular}




\begin{tabular}{|l|l|c|c|c|}
\hline \multirow{5}{*}{} & Systolic BP & $120.24 \pm 12.59$ & $124.72 \pm 12.80$ & 0.786 \\
\cline { 2 - 5 } & Diastolic BP & $75.79 \pm 9.63$ & $78.87 \pm 8.70$ & $0.041^{*}$ \\
\cline { 2 - 5 } & MAP & $82.38 \pm 9.03$ & $86.60 \pm 11.55$ & $0.014^{*}$ \\
\hline \multirow{5}{*}{$4^{\text {th }}$ day } & Heart rate & $89.98 \pm 12.74$ & $98.83 \pm 17.97$ & $0.004^{*}$ \\
\cline { 2 - 5 } & Respiratory rate & $25.80 \pm 3.12$ & $30.86 \pm 3.31$ & $0.002^{*}$ \\
\cline { 2 - 5 } & Temperature & $37.35 \pm 0.24$ & $38.44 \pm 0.30$ & $0.017^{*}$ \\
\cline { 2 - 5 } & Systolic BP & $119.34 \pm 10.62$ & $127.29 \pm 13.75$ & $0.033^{*}$ \\
\cline { 2 - 5 } & Diastolic BP & $73.93 \pm 9.64$ & $80.85 \pm 9.52$ & $0.015^{*}$ \\
\cline { 2 - 5 } $7^{\text {th }}$ day & MAP & $81.27 \pm 14.10$ & $88.73 \pm 11.69$ & $0.002^{*}$ \\
\hline \multirow{5}{*}{} & Heart rate & $88.55 \pm 14.56$ & $99.33 \pm 18.74$ & $0.023^{*}$ \\
\cline { 2 - 5 } & Respiratory rate & $23.06 \pm 3.19$ & $27.00 \pm 3.17$ & $0.007^{*}$ \\
\cline { 2 - 5 } & Temperature & $37.36 \pm 0.28$ & $38.48 \pm 0.78$ & $0.002^{*}$ \\
\cline { 2 - 5 } & Systolic BP & $119.72 \pm 12.98$ & $123.88 \pm 12.88$ & 0.147 \\
\cline { 2 - 5 } & Diastolic BP & $74.28 \pm 9.04$ & $77.76 \pm 8.96$ & $0.032^{*}$ \\
\cline { 2 - 5 } & MAP & $81.11 \pm 11.10$ & $85.61 \pm 10.98$ & $0.039^{*}$ \\
\hline
\end{tabular}

MAP: mean arterial pressure, Ns: There is no significant difference $(P>0.05) *$ Statistical significant difference $(p<0.05)$ hi-square test Independent samples $t$-test

Hypotheses (3) the expected mean arterial blood gases value of the study group will be better than those of the control group

Table (5): Comparison between the studied groups in relation to arterial blood gases \& oxygen saturation in both groups (after intervention)

\begin{tabular}{|c|c|c|c|c|c|c|c|}
\hline & \multirow{3}{*}{ Arterial blood gases } & \multicolumn{2}{|c|}{ Before intervention } & \multirow{3}{*}{ P-value } & \multicolumn{2}{|c|}{ After intervention } & \multirow{3}{*}{ P-value } \\
\hline & & Study $(n=60)$ & Control $(n=60)$ & & Study $(n=60)$ & Control $(n=60)$ & \\
\hline & & Mean \pm SD & Mean \pm SD & & Mean \pm SD & Mean \pm SD & \\
\hline \multirow{5}{*}{$1^{\text {st }}$ day } & PH & $7.14 \pm 0.12$ & $7.13 \pm 0.10$ & 0.625 & $7.30 \pm 0.48$ & $7.27 \pm 0.08$ & 0.598 \\
\hline & PaCo2 & $63.42 \pm 15.19$ & $63.20 \pm 25.51$ & 0.955 & $59.07 \pm 13.43$ & $60.93 \pm 16.61$ & 0.643 \\
\hline & $\mathbf{H C O 3}$ & $32.37 \pm 9.86$ & $32.47 \pm 7.61$ & 0.951 & $33.16 \pm 9.68$ & $32.84 \pm 7.95$ & 0.847 \\
\hline & $\mathrm{SaO2}$ & $88.95 \pm 7.49$ & $85.40 \pm 7.32$ & $0.012 *$ & $90.22 \pm 3.44$ & $88.21 \pm 4.92$ & $0.013^{*}$ \\
\hline & PaO2 & $54.16 \pm 21.76$ & $51.40 \pm 14.44$ & 0.420 & $53.53 \pm 15.82$ & $58.47 \pm 26.91$ & 0.229 \\
\hline \multirow{5}{*}{$2^{\text {nd }}$ day } & PH & $7.31 \pm 0.36$ & $7.30 \pm 0.06$ & 0.617 & $7.35 \pm 0.06$ & $7.30 \pm 0.07$ & $0.018^{*}$ \\
\hline & $\mathrm{PaCo2}$ & $56.36 \pm 11.37$ & $58.96 \pm 15.64$ & 0.459 & $52.75 \pm 17.80$ & $62.25 \pm 25.29$ & 0.264 \\
\hline & $\mathrm{HCO3}$ & $38.38 \pm 10.25$ & $36.31 \pm 7.28$ & 0.366 & $35.58 \pm 12.80$ & $32.95 \pm 6.75$ & 0.458 \\
\hline & \begin{tabular}{|l|}
$\mathrm{SaO2}$ \\
\end{tabular} & $90.25 \pm 3.65$ & $89.08 \pm 1.52$ & $0.024 *$ & $91.77 \pm 4.74$ & $90.65 \pm 2.12$ & $0.019^{*}$ \\
\hline & $\mathbf{P a O 2}$ & $73.00 \pm 9.35$ & $60.16 \pm 11.54$ & 0.318 & $86.33 \pm 9.92$ & $67.40 \pm 9.21$ & 0.015* \\
\hline \multirow{5}{*}{$3^{\text {rd day }}$} & PH & $7.32 \pm 0.06$ & $7.40 \pm 0.06$ & 0.022* & $7.38 \pm 0.06$ & $7.43 \pm 0.05$ & $0.028^{*}$ \\
\hline & PaCo & $57.47 \pm 12.37$ & $65.22 \pm 15.83$ & 0.169 & $54.67 \pm 10.87$ & $60.55 \pm 16.57$ & $0.049 *$ \\
\hline & $\mathrm{HCO3}$ & $33.44 \pm 9.08$ & $37.03 \pm 7.99$ & $0.048^{*}$ & $34.33 \pm 9.74$ & $35.09 \pm 10.12$ & $0.018^{*}$ \\
\hline & \begin{tabular}{|l|}
$\mathrm{SaO2}$ \\
\end{tabular} & $95.33 \pm 1.22$ & $92.89 \pm 1.18$ & $0.036^{*}$ & $94.50 \pm 3.70$ & $88.25 \pm 2.83$ & $0.001 *$ \\
\hline & PaO2 & $89.00 \pm 5.52$ & $71.21 \pm 8.36$ & $0.045^{*}$ & $87.75 \pm 14.86$ & $64.00 \pm 8.18$ & $0.005 *$ \\
\hline \multirow{5}{*}{$4^{\text {th }}$ day } & PH & $7.37 \pm 0.07$ & $7.40 \pm 0.09$ & $0.010^{*}$ & $7.39 \pm 0.07$ & $7.43 \pm 0.08$ & 0.020* \\
\hline & PaCo2 & $54.12 \pm 15.60$ & $58.46 \pm 20.16$ & 0.194 & $48.25 \pm 16.99$ & $58.85 \pm 13.14$ & $0.034 *$ \\
\hline & $\mathrm{HCO3}$ & $30.69 \pm 9.81$ & $34.00 \pm 8.11$ & 0.676 & $24.71 \pm 7.40$ & $32.23 \pm 6.73$ & 0.009 \\
\hline & SaO2 & $94.59 \pm 2.62$ & $92.93 \pm 3.77$ & $0.007 *$ & $95.50 \pm 2.39$ & $91.86 \pm 5.33$ & $0.005^{*}$ \\
\hline & $\mathbf{P a O 2}$ & $89.46 \pm 12.84$ & $76.07 \pm 13.22$ & $0.021 *$ & $95.00 \pm 9.46$ & $72.71 \pm 12.84$ & 0.020* \\
\hline \multirow{5}{*}{$7^{\text {th }}$ day } & PH & $7.41 \pm 2.07$ & $7.44 \pm 0.06$ & $0.029^{*}$ & $7.38 \pm 2.28$ & $7.45 \pm 0.05$ & $0.010 *$ \\
\hline & PaCo2 & $50.25 \pm 5.33$ & $55.84 \pm 11.80$ & $0.032 *$ & $43.00 \pm 4.35$ & $55.89 \pm 14.09$ & $0.007 *$ \\
\hline & $\mathrm{HCO3}$ & $26.78 \pm 4.86$ & $30.34 \pm 8.14$ & $0.024 *$ & $23.00 \pm 2.46$ & $28.11 \pm 5.84$ & 0.015* \\
\hline & $\mathrm{SaO2}$ & $94.03 \pm 4.34$ & $92.43 \pm 2.13$ & 0.003 & $97.00 \pm 2.83$ & $93.10 \pm 1.97$ & $0.002 *$ \\
\hline & $\mathbf{P a O 2}$ & $98.42 \pm 10.13$ & $79.86 \pm 10.83$ & 0.009 & $107.50 \pm 28.99$ & $83.10 \pm 10.03$ & 0.001* \\
\hline
\end{tabular}

Ns: There is no significant difference $(P>0.05) *$ Statistical significant difference $(p<0.05)$ ABG: Arterial blood gas, Paco ${ }_{2}$ : Partial pressure of carbon dioxide, $\mathrm{PaO}_{2}$ : Partial pressure of oxygen $\mathrm{Hco}_{3}$ : Bicarbonate $\quad \mathrm{SaO}_{2}: \mathrm{Oxygen}$ saturation Independent samples $t$-test 
Table (6): Comparison between the studied groups in relation to mechanical ventilator parameters.

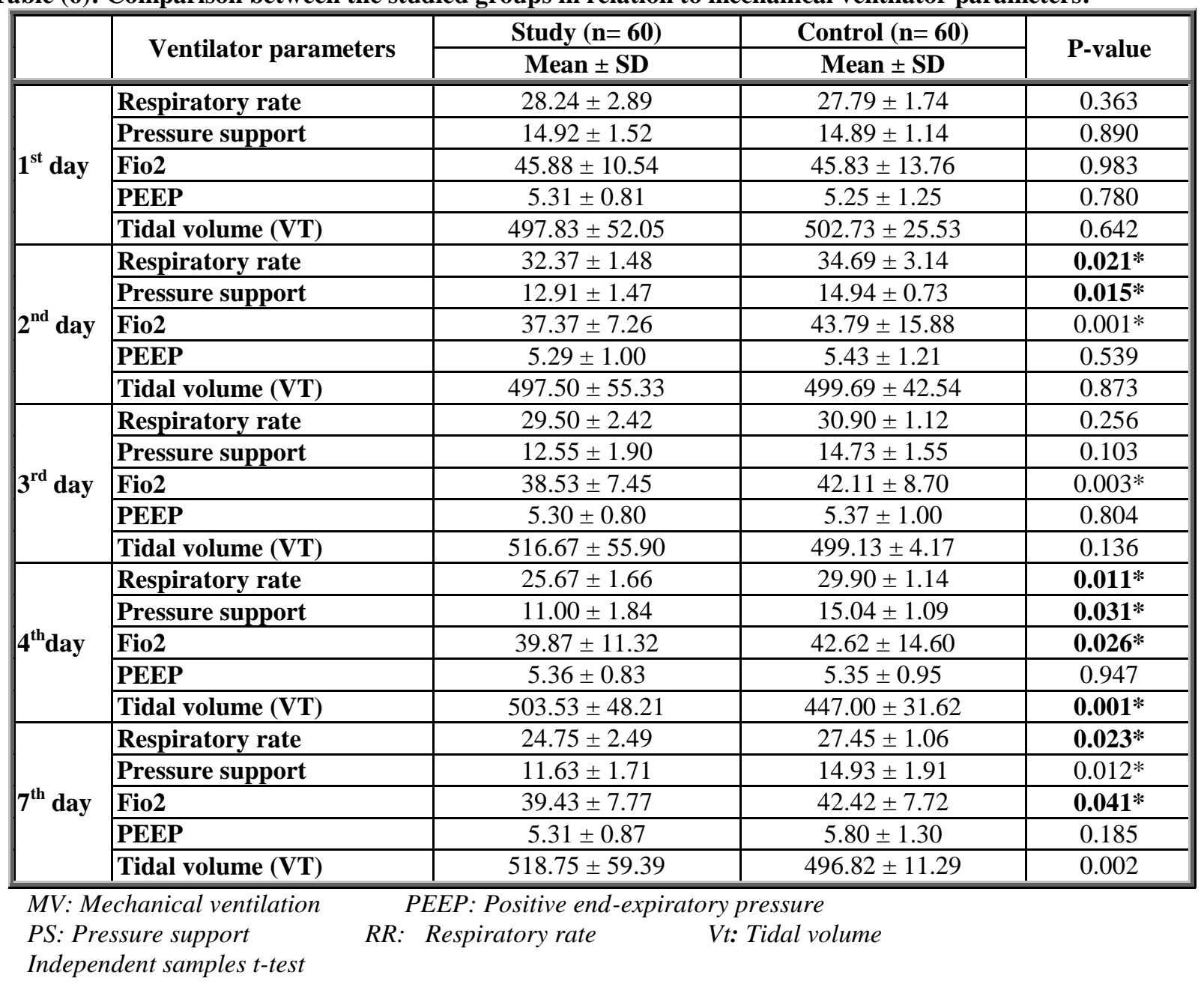

Table (7): percentage distribution of the studied patients regarding the finding of sputum culture.

\begin{tabular}{|l|c|c|c|c|c|}
\hline \multicolumn{1}{c}{ Variables } & \multicolumn{2}{c|}{ Study (n= 60) } & \multicolumn{2}{c|}{ Control (n= 60) } & \multirow{2}{*}{ P-value } \\
\cline { 2 - 7 } & No. & $\%$ & No. & \% & \\
\hline Finding of Sputum Cult. & & & & & \\
\hline Acentiobacter & 2 & 3.3 & 10 & 16.7 & $0.032^{*}$ \\
\hline E-coli & 3 & 5.0 & 9 & 15.0 & $0.023^{*}$ \\
\hline Klebsiella & 7 & 11.7 & 10 & 16.7 & 0.104 \\
\hline Staphylococcus & 5 & 8.3 & 13 & 21.7 & $0.041^{*}$ \\
\hline Pseudomonas & 0 & 0.0 & 5 & 8.3 & $0.006^{*}$ \\
\hline Streptococcus & 3 & 5.0 & 10 & 16.7 & $0.034^{*}$ \\
\hline No growth & 25 & 41.7 & 3 & 5.0 & $0.000^{*}$ \\
\hline
\end{tabular}

Ns: There is no significant difference $(P>0.05) *$ Statistically significant difference $(p<0.05)$

Chi-square test

Fisher Exact test

Hypotheses (4) the post mean clinical pulmonary infection reading of patients in the study group will be better than those of the control group. 
Table (8): Comparison between the study \& control group in relation to (Clinical Pulmonary infection Score).

\begin{tabular}{|c|c|c|c|c|c|c|}
\hline \multirow{2}{*}{ Days } & \multirow{2}{*}{ Variables } & \multicolumn{2}{|c|}{ G1 "study group "n=60 } & \multicolumn{2}{|c|}{$\begin{array}{c}\text { G2 "control group " } \\
n=60\end{array}$} & \multirow[t]{2}{*}{ P-value } \\
\hline & & $\mathbf{N}$ & $\%$ & $\mathbf{N}$ & $\%$ & \\
\hline \multirow{3}{*}{$1^{\text {st }}$} & No VAP & 52 & 86.7 & 45 & 75.0 & \multirow{2}{*}{0.104} \\
\hline & VAP & 8 & 13.3 & 15 & 25.0 & \\
\hline & Mean \pm S.D & \multicolumn{2}{|c|}{$5.67 \pm 1.971$} & \multicolumn{2}{|c|}{$6.37 \pm 2.125$} & \\
\hline \multirow{3}{*}{$4^{\text {th }}$} & No VAP & 42 & 70.0 & 27 & 45.0 & \multirow{2}{*}{$0.006^{*}$} \\
\hline & VAP & 18 & 30.0 & 33 & 55.0 & \\
\hline & Mean \pm S.D & \multicolumn{2}{|c|}{$5.70 \pm 2.152$} & \multicolumn{2}{|c|}{$6.83 \pm 2.291$} & \\
\hline \multirow{3}{*}{$7^{\text {th }}$} & No VAP & 51 & 85.0 & 17 & 28.3 & \multirow{3}{*}{$0.000^{*}$} \\
\hline & VAP & 9 & 15.0 & 43 & 71.7 & \\
\hline & Mean \pm S.D & \multicolumn{2}{|c|}{$4.53 \pm 1.167$} & \multicolumn{2}{|c|}{$7.30 \pm 2.003$} & \\
\hline
\end{tabular}

Ns: There is no significant difference $(P>0.05) *$ Statistically significant difference $(p<0.05)$

Chi-square test

Hypotheses (5) the post means patients outcomes reading of patients on the study group will be better than those of the control group.

Table (9): Percentage distribution of the studied patients regarding patient's outcome

\begin{tabular}{|c|c|c|c|c|c|}
\hline \multirow{2}{*}{ Variable } & \multicolumn{2}{|c|}{ Study $(n=60)$} & \multicolumn{2}{|c|}{ Control $(n=60)$} & \multirow{2}{*}{ P-value } \\
\hline & No. & $\%$ & No. & $\%$ & \\
\hline \multicolumn{5}{|l|}{ Mortality: } & \multirow{3}{*}{$0.020 *$} \\
\hline Survived & 46 & 76.7 & 34 & 56.7 & \\
\hline Died & 14 & 23.3 & $\mathrm{I} 26$ & 43.3 & \\
\hline \multicolumn{5}{|l|}{ Duration of mechanical ventilation: } & \multirow{4}{*}{$0.002^{*}$} \\
\hline$<5$ & 31 & 57.4 & 17 & 29.3 & \\
\hline $5-<10$ & 14 & 25.9 & 15 & 25.9 & \\
\hline$\geq 10$ & 9 & 16.7 & 26 & 44.8 & \\
\hline \multicolumn{5}{|l|}{ Duration of ICU stays: } & \multirow{4}{*}{$0.040^{*}$} \\
\hline$<5$ days & 15 & 25.0 & 4 & 6.7 & \\
\hline $5-<10$ days & 21 & 35.0 & 20 & 33.3 & \\
\hline$\geq 10$ days & 24 & 40.0 & 36 & 60.0 & \\
\hline Mean \pm S.D & \multicolumn{2}{|c|}{$9.22 \pm 6.00$} & \multicolumn{2}{|c|}{$12.63 \pm 8.62$} & $0.013^{*}$ \\
\hline \multicolumn{5}{|l|}{ Weaning: } & \multirow{3}{*}{$0.005^{*}$} \\
\hline Failure & 12 & 20.0 & 34 & 56.7 & \\
\hline Success & 48 & 80.4 & 23 & 43.3 & \\
\hline \multicolumn{6}{|l|}{ Complication: } \\
\hline Congestive heart failure & 11 & 18.3 & 34 & 56.7 & $0.000^{*}$ \\
\hline Renal Failure & 2 & 3.3 & 10 & 16.7 & $0.026^{*}$ \\
\hline Pneumothorax & 4 & 13.3 & 11 & 18.3 & $0.037 *$ \\
\hline Pulmonary edema & 5 & 8.3 & 12 & 20.0 & $0.039 *$ \\
\hline Abdominal distention & 19 & 31.6 & 36 & 60.0 & $0.000 *$ \\
\hline Pulmonary embolism & 6 & 20.0 & 18 & 60.0 & $0.002 *$ \\
\hline
\end{tabular}

Ns: There is no significant difference $(P>0.05) *$ Statistical significant difference $(p<0.05)$

Chi-square test Independent samples t-test 


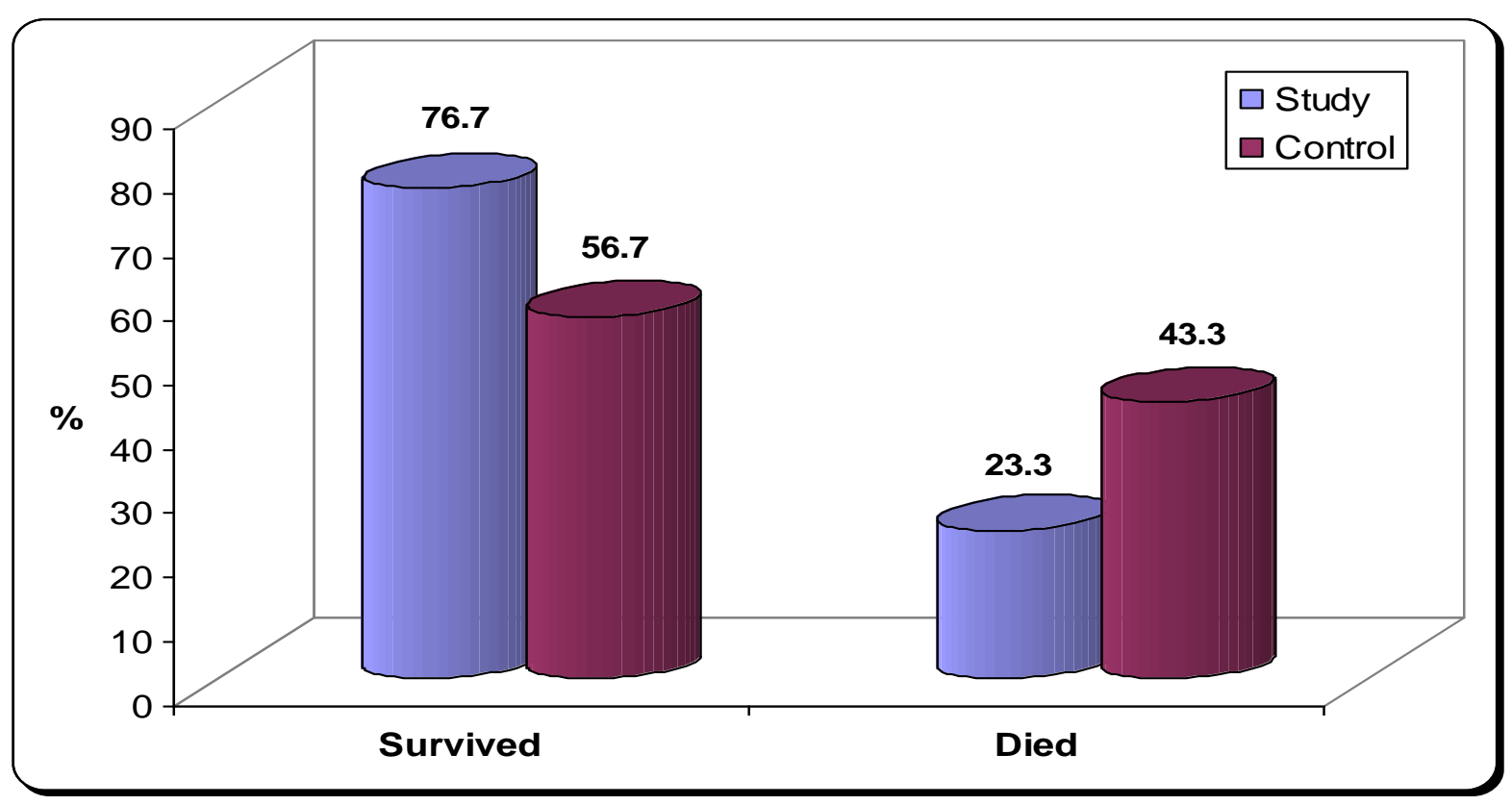

Figure (1): Percentage distribution of mortality rate of respiratory failure.

Table (1): It noticed that the mean age in the study group $(47.16 \pm 17.38)$ years and $(46.74 \pm 15.39)$ years. Also, there (40.0\%) of the sample in the control group were female versus $(38.3 \%)$ in the study group with no significant difference. While in relation to clinical data, it was founded that Type II of respiratory failure ( hypercapnic) most common in both groups

Table (2): Showed comparison between both groups in relation to breath sound the result revealed that there was nosignificant difference between the two groups in the $1 \mathrm{st}, 2^{\text {nd }}$ and $3^{\text {rd }}$ while significant improvement was shown in the study group in the $4^{\text {th }}, 7^{\text {th }}$ daycomparing with the control group.

Table (3): Showed comparison between both groups regarding the amount of tracheal secretion, the result revealed that highly significant difference with $(\mathrm{p}=0.008)$, which founded $(81.7 \%)$ of the study group had small amount versus $(96.7 \%)$ in the control group, and found (18.3\%) of the study group had moderate amount versus $(3.3 \%)$ in the control group in $1^{\text {st }}$ day. While in 2 nd daythere was nosignificant difference between the two groups, also, significant improvement in a study on the 4th and 7th days comparing with a control group with ( $\mathrm{p}=0.017 \&$ 0.006respectively) Thus, hypothesis (1) can be supported

Table (4): As regards hemodynamic parameters, it was founded that there was an increase in heart rate, body temperature, and respiratory rate among control group subjects comparing with the study group with a statistically significant difference $(p=<0.05)$. While regarding systolic and diastolic BP can report normotensive between both groups, and present a highly significant difference in the $2^{\text {nd }}, 4^{\text {th }}$ day. Also regarding mean arterial pressure can observe a statistically significant difference in the study group compared to the control group in $2^{\text {nd }}, 3^{\text {rd }} 4^{\text {th, }}$ and $7^{\text {th }}$.Thus, hypothesis(2), can be partially supported

Table (5): Showed comparison between the studied groups in relation to arterial blood gases, which the result after intervention according to the level of blood (PH) in 2,3, 4\& 7days observed statistical significance difference $(\mathrm{p}<0.05)$

Regarding the mean value of partial pressure of arterial oxygen $\left(\mathrm{PaO}_{2}\right)$, it was founded that statistical significance difference $(\mathrm{p}<0.05)$ in $2^{\text {nd }}, 3^{\text {rd }}, 4^{\text {th }}$, and 7 days in the study group when compared with the control group. Finally, the result of the partial pressure of arterial oxygen $(\mathrm{PaO} 2)$ in the study group was better than the control group after intervention.

Regarding the concerning the partial pressure of arterial carbon dioxide $\left(\mathrm{PaCO}_{2}\right)$ it was reported that significance reduction $\mathrm{PaCo}_{2}$ among study group when compared with the control group after intervention.

As regarding the level of bicarbonate $\left(\mathrm{HCO}_{3}\right)$ in blood, the result revealed significant improvement between both groups from $3^{\text {rd }}$ day Thus, hypothesis (3), partially can be supported

Table (6): Showed a comparison between the studied groups in relation to mechanical ventilator parameters which the result reported to mean value of tidal volume (VT)among patients in both groups. there 
presents a highly significant difference in 4th and 7th day with $(\mathrm{p}=0.001 \& 0.002$ respectively ). While regarding $\mathrm{FiO} 2$ the results of the current study reflect that percentage of oxygen was utilized among the whole study sample it was founded that high $\mathrm{FIO}_{2}$ was needed in the control group, and there was a statistical significance difference $(p<0.05)$ in $2^{\text {nd }}$ $3^{\text {rd }}, 4^{\text {th }} \& 7^{\text {th }}$ days. Also regarding pressure support (PS) show, there was significance in all days except $1^{\text {st }}$. In relation to positive end-expiratory pressure (PEEP), there is no significant difference founded in $1^{\text {st }}, 2^{\text {nd }}, 3^{\text {rd }}, 4^{\text {th }} \& 7^{\text {th }}$ days.

Table (7): Show comparison between both study \& control groups regarding the finding of sputum culture, the result revealed that most common bacterial sputum cultures were Staphylococcus, Streptococcus, Acinetobacter, Klebsiella, and Pseudomonas. Also, the result revealed that present significant difference between both groups in all items except Klebsiella

Table (8): Show a comparison between the study \& control group in relation to (Clinical Pulmonary infection Score). the result showed a highly statistically significant difference between study and control group with $(\mathrm{P}=0.005)$. in the $4^{\text {th }}$ and $7^{\text {th }}$ days and a higher percentage of VAP was observed among the control group.

Table (9): Showed Percentage distribution of the studied patients regarding the patient's outcome the result revealed the incidence of the mortality rate was a statistically significant difference $\left(\mathrm{p}=0.020^{*}\right)$ when compared study group to control group.

As regarding the duration of mechanical ventilation, the result showed a highly significant difference between study and control groups ( $\mathrm{P}=0.002)$. While regarding Weaning frommechanical ventilation the result showed a highly significant difference between study and control group $(\mathrm{P}=0.005)$.

As regarding the duration of ICU stay, the result showed that the mean valve of ICU stays in the study group lower than that of the control group $(9.22 \pm$ $6.00 \& 12.63 \pm 8.62$ respectively with a statistically significant difference.

While regarding the occurrence of complication, it was observed that congestive heart failurepulmonary embolism and abdominal distention were most common complication occurred among control group subjects. Also, a highly statistically significant difference regarding all items of complication, when compared study group with the control group.

Fig (1): Showed percentage distribution of the studied patients regarding 1patient's outcome the result revealed the incidence of the mortality rate was a highly significant difference $\left(p=0.020^{*}\right)$ when compared study group to control group.

\section{Discussion}

In acute respiratory failure, ventilatory support is used for totally or partially replaces spontaneous ventilation. So, caring for a mechanically ventilated patient considered the core of nursing care in ICU. Where nurse's responsibility is to maintain the permeability of the intubated patient's airway areas, as well as the mastery over ventilator parameters, necessary to assess patient's adaptation to the parameters, promote patient safety and provide good services dos Santos et al., (2014).

Chang et al., (2017) reported that Providing comprehensive nursing services to patients (e.g., psychological, diet, oxygen interventions, sputum excretion, oral and environmental cleaning) relieves the clinical symptoms of patients and creates a comfortable therapeutic environment for the patients, improves the treatment experience of the patients, ensures their safety, and increases their degree of satisfaction

According to age the results of the present study revealed that mean age in study and control groups was around fifty years with subjects no statistically significant differences. This not agreed with Nafae (2018) who evaluated the effect of multimodal physiotherapy on the outcome of forty mechanically ventilated patients diagnosed with acute respiratory failure and reported that the mean age of patients around fifty years old, with no significant difference. Regarding gender, our study showed that a higher percentage of patients in both groups were male which not agreed with the study of Gadre et al., (2018) about"Acute respiratory failure requiring mechanical ventilation in severe chronic obstructive pulmonary disease (COPD). Who reported that about half were male.

Osadnik et al., (2017) in a study about" Noninvasive ventilation for the management of acute hypercapnic respiratory failure due to exacerbation of chronic obstructive pulmonary disease". Reported that most participants from a male with age more than sixty years.

Regarding clinical data of patients. The result of the present study showed that: the majority of studied patients in the chest ICU were typing II ARF. This match to the result of the study done by (Garrouste et al., 2018) who mentioned that hypercapnic respiratory failure (type 2) in which $\mathrm{paco}_{2}>50 \mathrm{mmHg}$, is a result of scant alveolar ventilation subordinate to decreased ventilator drive, respiratory muscle fatigue or failure and increased work of breathing.

Regarding breath sound, the study found that the majority of patients in both groups hadRonchi with no significant difference between both groups in the first day. More than half of subjects in the study group had normal breathing sounds in the four \&seven days 
which reflect the effect of the nursing intervention( chest physiotherapy, nebulization, suctioning) and medical treatment versus the control group with significant differences.

Regarding the number of tracheal secretions, our study revealed that highly significant difference, which founded most of the study group had small amount versus majority in the control group, and found little number of the study group had moderate amount versus in the control group in $1^{\text {st }}$ day. While on the 2nd daythere was nosignificant difference between the two groups, also, significant improvement in the study on the 4th and 7th days comparing with the control group .

Smeltzer \& Bare (2010) emphasized that retained secretions are a threat to a mechanically ventilated patient. If audible crackles are present, it is necessary to use chest percussion with cough routine until the lungs are clear. Nurses should listen to both lungs, anteriorly and posteriorly, to determine whether there are any changes in breath sounds, diminished breath sounds, monitor sputum color, consistency, odor, and amount also evaluate chest $\mathrm{x}$-ray.

This is the same line with Tatham \& Patel, (2018) who found that the clinical examination for patients who developed in acute respiratory failure shortly after admission in ICU, auscultation revealed inspiratory Ronchi in both lower lobes that despite severe cases.

Our results are agreed with findings of Wang, et al., (2018) who evaluated the effect of chest physiotherapy with early mobilization in about five hundred critically ill patients in the Intensive care units and reported that chest physiotherapy (CPT) help patients in reducing the accumulation of airway secretion and preventing collapsed lung. AbuBakr, et al., (2018) in a study about "Effect of Pulmonary Care measures on Reducing Respiratory Tract Infection and Dispend Grades among Postoperative Elderly Patients with Abdominal Surgeries"." reported that patient in the study group showed significant improvement breathing sound from 3rd day compared with a control group.

A randomized, single-blind experimental study conducted by Chen et al., (2009) in about hundred patients were enrolled from 2 ICUs and randomly assigned into either the experimental group or control group, while patients in the control group received routine positioning care, which consisted of a change in body position every 2 hours. Patients in the experimental group received routine positioning care plus the use of chest vibration nursing intervention experimental group showed that the best result for sputum excretion after the chest vibration intervention.
Hariedy, et al., (2015) revealed that, highest number of patient who receives regular CPT \& RM had a large number of secretions while patients who receive routine nursing care gave a moderate amount of secretions \& there was a highly statistically significant difference between both groups. This difference is due to the effect of chest physiotherapy which mobilizes the respiratory secretions from central to peripheral airway \& increases the amount of tracheobronchial mucus cleared from the respiratory tract.

with (Negro et al., 2016) result who mentioned that most of the patients with respiratory failure had a large and high amount of tracheal secretion with suction this in relation to inability of them to discharge these secretions in addition to the incidence of infection.

Muhammad, et al., (2014) evaluated the effect of Chest Physical Therapy on sixty patients diagnosed with bronchiectasis, reported that this intervention had a significant effect on airway clearance, reduced recurrence, and improvement in dyspnea, breath sounds, PEFR, reduction in sputum quantity and improvement in $\mathrm{SpO} 2$.

Regarding vital signs and hemodynamic parameters, it was noticed that there was an increase in heart rate, body temperature, and respiratory rate among control group subjects comparing with the study group with a statistically significant difference. While regarding systolic and diastolic BP can report normotensive between both groups, and present a highly significant difference in the $2^{\text {nd }}, 4^{\text {th }}$ day. Also regarding mean arterial pressure can observe a statistically significant difference in the study group compared to the control group in $2^{\text {nd }}, 3^{\text {rd }}{ }_{4}^{\text {th, }}$ and $7^{\text {th }}$.

Our study compatible with Long et al., (2018) who evaluated the effects of nursing intervention on patients with respiratory diseases reported that intervention group showed lower heart rate and respiratory rate compared with the control group with highly statistically significant differences .

(Cortes-Puentes, et al., 2018) monitoring of hemodynamics is crucial when aiming simultaneously to optimize perfusion, improve gas exchange, and minimize VILI in patients with ARDS and hemodynamic failure.

Zeng, et al., (2017) investigated the effect of chest physiotherapy (CPT) on patients sixty eight undergoing mechanical ventilation (MV). Where thirty seven patients received comprehensive chest physiotherapy while The patients in the control group (thirty one patients) received routine hospital care; There was no significant difference in vital signs parameters excepting pulse oxygen saturation $(\mathrm{SpO} 2)$ was significantly higher than that control group. 
Our study showed an increase in heart rate, temperature, respiratory rate, and abnormal breathing sound in addition to positive sputum culture in more than two-thirds of the control group which indicates the presence of chest infection. Which was mentioned by Moore et al., 2017) in a study about "Predictors of pneumonia in lower respiratory tract infections prospective cough complication " that the best current diagnostic model for pneumonia for use in a primary care setting includes an absence of runny nose and presence of breathlessness, crackles, and diminished breath sounds on auscultation, tachycardia and fever .

Regarding arterial blood gas, it was noticed statistical significance difference between both groups related to $\mathrm{PH}, \mathrm{PaO}_{2}, \mathrm{Paco}_{2}, \mathrm{Hco}_{3}$ and $\mathrm{SaO}_{2}$ in after intervention in the study group compared with the control group Our results revealed an increase in paco2, decreased in ph, and elevation in bicarbonate in the first day which supports our finding that acute respiratory failure type II was the most common among subjects in both groups which matched with (Morton \& Fontaine 2018), who reported that in acute respiratory failure II (hypercapnia), arterial blood $\mathrm{pH}$ is decreased, which means acute respiratory acidosis. Patients with hypercapnia may exhibit an acute elevated in $\mathrm{PaCO} 2$ to a high level, a decrease of blood $\mathrm{pH}$, and a significant increase in serum bicarbonate during the onset of acute respiratory failure.

This compatible with findings of Santos, et al., (2014) evaluated the immediate effects of chest physiotherapy on hemodynamic, metabolic, and oxidative stress parameters in subjects with septic shock. they founded a significant increase in arterial $\mathrm{pH}, \mathrm{PaO} 2$, and arterial oxygen saturation and also a significant reduction in $\mathrm{PaCO} 2$ after physiotherapy.

The study done by (Funk et al., 2013) mentioned that, the patients suffer from ARF had hypercapnia episodes similar efficacy as for subjects with COPD. In addition, (Lun et al., 2016) founded in their study; A proportion of patients with more severe disease will have respiratory acidosis as a result of acute on chronic respiratory failure. Acidosis is associated with increased mortality and also a higher need for intubation.

Regarding mechanical ventilator parameters, the result reported to mean the value of tidalvolume (VT)among patients in both groups. there presents a highly significant difference in 4th and 7 th day respectively ). While regarding $\mathrm{FiO} 2$ the results of the current study reflect that percentage of oxygen was utilized among the whole study sample it was founded that high $\mathrm{FIO}_{2}$ was needed in the control group, and there was a statistical significance difference in $2^{\text {nd }} 3^{\text {rd }}, 4^{\text {th }} \& 7^{\text {th }}$ days. Also regarding pressure support (PS) show, there was significance in all days except $1^{\text {st }}$. In relation to positive endexpiratory pressure (PEEP), there is no significant difference founded in $1^{\text {st }}, 2^{\text {nd }}, 3^{\text {rd }}, 4^{\text {th }} \& 7^{\text {th }}$ days.

Pattanshetty \& Gaude 2011 in their study about " Effect of multimodality chest physiotherapy on the rate of recovery and prevention of complications in patients with mechanical ventilation" demonstrated significant improvements in the study group as regard to ventilator parameters such as Tidal volume, PEEP and lung compliance between both the groups.

Similarly, on the contrary, the Gajic study found higher airway pressures (peak inspiratory, plateau, and driving pressure), lower PEEP, and higher respiratory rate accompanying with higher hospital mortality, along with older age and $\operatorname{ARF}($ Gahic et al., 2011).

As regarded sputum culture the result revealed that present highly significant difference between both groups according to Acinetobacterwith. Also, a significant difference according to E-coliand found a highly significant difference according to Klebsiella $(p=0.003)$. While regarding Staphylococcus that observed statistically significant differences between both groups $(\mathrm{p}=0.041)$. While according to Pseudomonas the result revealed that a highly significant difference was found .Also Streptococcus that observed a statistically significant difference between both groups $(\mathrm{p}=0.009)$

Duggan et al., (2010) emphasized that retained airway secretions occlude the airways of intubated and mechanically ventilated patients and the persistent presence of sputum in the airways provide an ideal environment for colonizing organisms.

Our study showed that more than two-thirds of the control group had positive sputum culture and about one third had negative culture after applying of chest physiotherapy this is matched with Abd El-Hamid et al., (2017) Demonstrates the microbiological findings of ETT cultures after CPT care positive microbiology findings denoting VAP were found in $40 \%$ of patients in the control group and $20 \%$ of patients in the study group. The difference was statistically significant between the two groups.

Boten, (2010) added that prophylactic chest physiotherapy should be initiated to prevent respiratory complications rather than to wait until atelectasis or retained secretion occurs, turning to cough and breathing exercises should be started at least every 2 hours, deep breathing should be encouraged more frequently.

Regarding clinical pulmonary infection score, in our study, it was noticed that mean CPIS in the control group higher than that of the study group which indicates a higher incidence of VAP among the control group.and lower among study groups due to the effect of chest physiotherapy on clearance of 
secretions from the respiratory tract so preventing colonization of microorganisms and chest infection. This result agrees with Abd El-Hamid et al., (2017) demonstrates the mean CPIS after chest physiotherapy care in the study group lower than that in the control group with significant difference. Aysha et al., (2016) in a study about" Efficacy of implementing nursing care protocol on the incidence of VAP in ICU at Tanta Emergency Hospital" showed that who found that, the occurrence of VAP according to clinical pulmonary infection score among automatically ventilated suffers was $75 \%$ in the control group compared to $35 \%$ in a study group with $\mathrm{p}=0.001$.Regarding patients outcomes, our study noticed that majority of patients in the study group were survived versus the majority died in the control group. While regarding the duration of mechanical ventilation, it was found that half of the patients in the control group were $\geq$ ten days versus more than a third of patients in the study group were <five days. Regarding Occurrence of complications, it was noticed that the majority of patients in the control group was complicated versus just half of the patients in the study group As regarding duration of ICU stay, the result showed that mean valve of ICU stay in study group lower than that of the control group with a statistically significant difference.

Castro (2013) compared at least four daily CPT Sessions with one CPT visit over 6 hours in 146 patients, 73 in each group. CPT comprised body positioning, manual chest percussions, and suctioning in both groups. More intensive CPT resulted in a significantly shorter duration of ventilation and ICU stay, less respiratory infections, and lower mortality.

Meawad et al., (2018) in a study about "effect of Chest Physical Therapy Modalities on Oxygen Saturation and Partial Pressure of Arterial Oxygen in Mechanically Ventilated Patients " The results support the importance of adding chest physiotherapy program to early MV patients as it decreases complications in patients undergoing MV, decreases ICU duration.

(Matkovic et al., 2012) Found that; the in-hospital mortality of patients hospitalized for ARF is 2-8\%( up to $15 \%$ for ICU patients). (Morton \& Fontaine 2018), acute respiratory failure may be responsible for as many as $10 \%$ to $15 \%$ of admissions to medical ICUs and for as many as $50 \%$ to $75 \%$ of those patients who require ICU hospital stays longer than 7 days. (Fujishima et al., 2016) found in the outcomes of patients that $62 \%$ of patients suffered from renal insufficiently, but in our study, the majority of the studied sample died and others survived and suffered from heart failure.

\section{Conclusion}

Based on the findings of the present study, it can be concluded that nursing care for acute respiratory failure by Applying regular chest physiotherapy for in the chest care unit significantly improves respiratory parameters.

\section{Recommendation}

We can recommend that

- Develop standardized nursing guidelines for patients with acute respiratory failure.

- Provide a database that can be utilized by the health team members to raise nurse awareness and initiate more active nurse's role in chest ICU care unit.

- Develop educational program for nursing staff caring for patients with acute respiratory failure. Health professionals can utilize such information in the provision of care for acute respiratory failure patients in the future. Chest physiotherapy must be applied as a routine care for cases of acute respiratory failure admitted to the chest care unit to improve patient outcomes.

\section{References}

1. Abd El-Hamid G., Shams, Abd El-moaty A., Shaimaa Ahmed Awad S., (2017): "Effect of Using Different Modalities of Chest Physiotherapy on Prevention of VentilatorAssociated Pneumonia." IOSR Journal of Nursing and Health.Science (IOSR-JNHS), vol. 6, no. 5, 2017, pp. 19-35

2. AbuBakr, Ibrahim H., Tohamy T., Mohammed I., Mohamed J., (2018): "Effect of Pulmonary Care measures on Reducing Respiratory Tract Infection and Dispend Grades among Postoperative Elderly Patients with Abdominal Surgeries"." IOSR Journal of Nursing and Health Science (IOSR-JNHS), vol. 7, no.4, 2018, pp. 87-97.

3. Alcibey A., \& Isabel A., (2016): Metabolic Functions of the lung. Disorder and Associated Pathologies, Journal of clinical medicine research, 8(10):689-700.

4. Ames N., Sulima P., Yates J., \& McCullagh L., Gollins S., (2011): Effects of systematic oral care in critically ill patients: a multicenter study. Am J Crit Care 20: e103-114.

5. Aysha Z., Alaa El-din S., Attia N., \& Akab M., (2016): Efficacy of implementing nursing care protocol on the incidence of VAP in ICU at Tanta Emergency Hospital. Journal of American science; 12(2).

6. Bonten M., Gaillard C., \& van Tiel, F., (2010): Prevention of hospital-acquired 
pneumonia(the stomach is not a source for colonization of the upper respiratory tract and pneumonia in ICU patients.): Chest Am;17(4):773-84.

7. Castro A., Calil S., Freitas S., Oliveira A., Porto E., (2013): Chest physiotherapy effectiveness to reduce hospitalization and mechanical ventilation length of stay, pulmonary infection rate, and mortality in ICU patients. Respir Med. Jan; 107(1):68-74.

8. Chen Y., Wu L., Mu P., Lin L., Chou S., Shie H., (2009): Using chest vibration nursing intervention to improve expectoration of airway secretions and prevent lung collapse in ventilated ICU patients: a randomized controlled trial. J Chin Med Assoc.;72(6):316322.

9. Cortes-Puentes, G., Oeckler, R., \& Marini, J., (2018): Physiology-guided management of hemodynamics in acute respiratory distress syndrome.Annals of translational medicine, 6(18), 353.

10. Culnan, D., Sherman, W., Chung, K., \& Wolf, S., (2018): Critical Care in the severely Burned: Organ Support and Management of Complications. In Total Burn Care (pp. 328354) Elsevier.

11. dos Santos, R., Donadio, M., da Silva, G., Blattner, C., Melo, D., Nunes, F., \& de Oliveira, J., (2014): Immediate effects of chest physiotherapy on hemodynamic, metabolic, and oxidative stress parameters in subjects with septic shock. Respiratory care, 59(9), 1398-1403.

12. Duggan M., KavanagFahy J., \& Dickey B., (2010): Pulmonary atelectasis: a pathogenic perioperative entity. N Engl J Med, 363:22332247

13. Elizabeth G., \& Deborah M., (2018): Principles of systemic assessment, Children and Young Peoples Nursing Skills at a Glance, John Wiley\&Sons, pp 6-8.

14. Fujishima, S., Gando, S., Daizoh, S., Kushimoto, S., Ogura, H., Mayumi, T., \& Takeyama, N., (2016): Infection site is predictive of outcomes in acute lung injury associated with severe sepsis and septic shock, Respiratory, 21(5), 898-904.

15. Funk G., Bauer P., Burghuber O., Fazekas A., Hartl S., \& Hochrieser H., (2013): Prevalence and prognosis of COPD in critically ill patients between 1998 and 2008 . EurRespir J 2013;41(4):792-799.

16. Gadre S., Duggal A., \&Mireles-Cabodevila E., (2018): Acute respiratory failure requiring mechanical ventilation in severe chronic obstructive pulmonary disease (COPD). Medicine (Baltimore); 97(17):e0487.

17. Garrouste- Orgeas, M., Azoulay, E., Ruckly, S., Schwebel, C., de Montmoollin, E., Bedos, J., \&Dumenil, A., (2018): Diabetes was the only comorbid condition associated with mortality of invasive pneumococcal infection in ICU patients: a multicenter observational study from the Outcomerea research group. Infection, 46(5), 669-677.

18. Gil Wayne, (2016): Ineffective Tissue Perfusion, decrease in oxygen, resulting in failure to nourish tissues at the capillary level, Nurseslabs/Ut in Omnibus Glorificetur Deus.

19. Hariedy N., Mohamed W., Mohamed M., Abdel Aziz M., \& Morsy K., (2015): Chest Physiotherapy and Recruitment Maneuvers: Effects on Lung Mechanics and Pulmonary Complications among Mechanically Ventilated Patients with acute lung injury.IOSR Journal of Nursing and Health Science (IOSR-JNHS) e-ISSN: 2320-1959.pISSN: 2320-1940 Volume 4, Issue 4 Ver. II (Jul. -Aug. 2015), Pp 17-32 www.iosrjournals.org.

20. Higginson, R., \& Jones, B., (2009): Respiratory assessment in critically ill patients: airway and breathing. British Journal of Nursing, 18(8), 456.

21. Kaplow, R., Hardine S., (2012): Critical care nursing synergy for optimal outcomes USA, $8^{\text {th }}$ edition chapter 21 respiratory monitoring pp 274-290.

22. Lea F., Mauricio F., Mauricio F., Iuri C., Janete .S, Siliva R., Alvaro V., \& Mary C., (2016): Mechanical ventilation in patients in the intensive care unit of a general university hospital in southern Brazil: an epidemiological study, Clinical, 71(3):145-151.

23. Long G., Yue L., Peng Z., Xiong G., \& Li Y., (2018): Clinical effects of nursing intervention on severe patients in the respiratory department.Research Article Biomedical Research (2018) Volume 29, Issue 5.

24. Lun, C., T sui, M., Cheng, S., Chan, V., Leung, W., Cheung, A., \& C. M., (2016): Difference in baseline factors and survival between normocapnia, compensated respiratory acidosis and decompensated respiratory acidosis in COPD exacerbation: a pilot study. Respirology, 21(1), 128-136. 4358). CRC Press.

25. Matkovic Z., Huerta A., \& Soler N., (2012): Predictors of adverse outcomes in patients 
hospitalized for exacerbation of the chronic obstructive pulmonary disease. Respiration 2012; 84: 17-26.

26. Meatwad M., Abd El Aziz A., Obaya H., Mohamed S., Mounir K., (2018): effect of Chest Physical Therapy Modalities on Oxygen Saturation and Partial Pressure of Arterial Oxygen in Mechanically Ventilated Patients The Egyptian Journal of Hospital Medicine Volume 72, Issue 8 Summer 2018, Page 50055008.

27. Meg G., \& Judith L., (2017): Impaired gas exchange, Nursing Care Plan, Diagnoses, Intervention, and Outcomes, $9^{\text {th }}$ ed, Elsevier, $p p$ 80-82.

28. Mirza, S., Clay, R., Koslow, M., \& Scanlon, P., (2018, October): COPD guidelines: a review of the 2018 GOLD report. In Mayo Clinic Proceedings (Vol. 93, No. 10, pp. 14881502).Elsevier.

29. Moore M., Stuart B., Little P., (2017): Predictors of pneumonia in lower respiratory tract infections: 3C prospective cough complication cohort study. EurRespir J 2017; 50: 1700434

30. Morton G., \& Fontaine K., (2018): Critical care nursing a holistic approach, Critical care nursing a holistic approach - 10th edition 2018chappter 26 common respiratory disorder part 6 respiratory system p.p 582-599.

31. Muhammad, A., \& Bashir, M., \&Noor, R., (2014): effectiveness of chest physiotherapy in the management of bronchiectasis. Annals of KEMU. 20

32. Nafae R., El-Shahat H., Shehata S., Zaki L., (2018): effect of multimodal physiotherapy on the outcome of mechanically ventilated patients at zagazig university respiratory intensive care unit in (2014-2015) Z.U.M.J.Vol. 24; No.3.

33. Nancy D., Cliesla (2014): Chest physical therapy for patients in the Intensive care units journal of American physical therapy p.p 76: 609- 625.

34. Negro, A., Greco, M., \& Cabrini, L., (2016): Medical and Nursing Management of Tracheostomy. In Percutaneous.Tracheostomy in Critically Ill patients (pp.199130).Springer.Cham.

35. Osadnik, C., Tee, V., Carson, Chahhoud, K., Picot, J., Wedzicha, J., \& Smith, B., (2017): Non, invasive ventilation for the management of acute hypercapnia respiratory failure due to exacerbation of the chronic obstructive pulmonary disease. Cochrane Database of Systemic Reviews, (7).
36. Pattanshetty R., Gaude G., (2011): Effect of multimodality chest physiotherapy on the rate of recovery and prevention of complications in patients with mechanical ventilation: A prospective study in medical and surgical intensive care units. Indian J Med Sci;65:17585.

37. Santos Cleverson dos, Nascimento, Eliane Regina Pereira do, Hermida, PatríciaMadalena Vieira, Silva, Tatiana Gaffuri da, Galetto, Sabrina Guterres da Silva, Silva, Nelson Junior Cardoso da, \& Salum, NádiaChiodelli. (2020).Good nursing practices towards patients on invasive mechanical ventilation in hospital emergency.Escola Anna Nery, 24(2), e20190300.

38. Smeltzer, S., Bare, B., Hinkle J., \& Cheever K., (2010): Brunner and Suddarth's textbook of Medical-Surgical Nursing, $12^{\text {th }}$ ed., Wolters Kluwer Health/ Lippincott Williams \& Wilkins, vol. (1), unit (5), ch: (21),p:487.

39. Struik F., Sprooten R., Kerstjens H., Bladder G., Zijnen M., (2018): Nocturnal non- invasive ventilation in COPD patients with prolonged hypercapnia after ventilator support for acute respiratory failure: a randomized, controlled, parallel-group study, Non- invasive ventilation, BMJ Publishing Group, 69(9).

40. Tatham, K., \& Patel, K., (2018): Examination: Respiratory. Complete OSCE Skills for Medical and Surgical Finals (pp. 4358). CRC Press

41. Wang, T., Wu, C., \& Wang, L., (2018): Chest physiotherapy with early mobilization may improve extubation outcome in critically ill patients in the intensive care units. The clinical respiratory journal, 12(11), 26132621.

42. Zeng, H., Zhang, Z., Gong, Y., \& Chen, M., (2017): Effect of chest physiotherapy in patients undergoing mechanical ventilation: a prospective randomized controlled trial. ZhonghuaWeiZhong Bing Ji Jiu Yi Хие, 29(5), 403-406 